\title{
Pulsed Nd:YAG laser marking system for wood surfaces
}

\section{Sistema de laser Nd:YAG pulsado para marcado de superficies de madera}

\author{
H. H. Cerecedo-Núñez ${ }^{(1, *}$, A) , J. B. Benítez-Moreno(2), P. Padilla-Sosa(1, A) \\ 1. Facultad de Física e Inteligencia Artificial, Universidad Veracruzana, Xalapa, Ver., México. \\ 2. Facultad de Ingeniería en Instrumentación Electrónica, Universidad Veracruzana, Xalapa, Ver. México. \\ (*)Email: hcerecedo@uv.mx \\ A: miembro de AMO / AMO member \\ Recibido / Received: 10/01/2012. Revisado / Revised: 14/07/2012. Aceptado / Accepted: 16/07/2012. \\ DOI: http://dx.doi.org/10.7149/OPA.45.3.369
}

\begin{abstract}
:
This work reports the development of a complete laser marking system applied to study wood surface marking. In this development, the output pulses of light from a Nd:YAG laser are controlled by positioning them in a desired place and time, according to the image to be printed. The entire process is automated through an interconnection between the laser, a microcontroller and a computer. The main contributions of this work are the use of a specifically pulsed laser, the integration of all parts involved in the marking process and the marking study over a wood surface.
\end{abstract}

Key words: Optical Marking, Pulsed Lasers, Virtual Instrumentation.

\section{RESUMEN:}

En este trabajo se reporta el desarrollo de un sistema de marcado láser, aplicado al estudio de marcado sobre superficies de madera. Con esto, los pulsos de un láser son controlados adecuadamente para ubicarlos en una posición y tiempo deseados, de acuerdo con la imagen que se desee imprimir. El proceso completo es automatizado por medio de una interconexión entre el laser, un micro-controlador y una computadora. La principal contribución de este trabajo es el empleo de un láser específico, la integración de todas las partes involucradas en el proceso de marcado y el empleo de ello sobre superficies de madera.

Palabras clave: Marcado Láser, Láser Pulsado, Instrumentación Virtual.

\section{REFERENCIAS Y ENLACES / REFERENCES AND LINKS}

[1]. X.-S. U. Yi, Y. Feng, "Etching behavior of plastics using a near infrared laser", J. Mater. Sci. Lett. 18, 245247 (1999).

[2]. Y. Hanada, K. Sugioka, Y. Gomi, H. Yamaoka, O. Otsuki, I. Miyamoto, K. Midorikawa, "Development of practical system for laser-induced plasma-assisted ablation (LIPAA) for micromachining of glass materials", Appl. Phys. A - Mater. 79, 1001-1003 (2004)

[3]. S. Bednarczyk, R. Bechir, P. Baclet, "Laser micro-machining of small objects for high-energy laser experiments", Appl. Phys. A - Mater. 69, S495-S500 (1999).

[4]. T. Otani, L. Herbst, M. Heglin, S. V. Govorkov, A. O. Wiessner, "Microdrilling and micromachining with diode-pumped solid-state lasers", Appl. Phys. A - Mater. 79, 1335-1339 (2004).

[5]. Cobra diode pumped Nd:YAG laser marking systems, Electrox (2004). www.electrox.com

[6]. P. Grace, The Art of Laser Marking, Application Report, Industrial Laser Solutions Magazine, August (2006).

[7] J. Hecht, D. Teresi, Laser, Supertool of the 1980s, Ticker \& Fields, 383 Orange Street, New Heaven, Connecticut 06511, USA (1982). 
[8]. Handbook of Laser Technology and Applications, Volume III: Laser Design and Laser Systems, C. E Webb, J. D C Jones Edt. IOP Publishing Ltd (2004).

[9]. B. Rethfield, K. Sokolowski-Tinten, D. Von Der Linde, S. L. Anisimov, "Timescales in the response of materials to femtosecond laser excitation", Appl. Phys. A-Mater. 79, 767-769 (2004).

[10].M. Beck, F. Dausinger, H. Hiigel, "Studie zur energieeinkopplung beim tiefschweiben mit laserstrahlung", Laser Optoelektron. 21, 80 (1989).

[11].P. W. Fuerschbach, "Measurement and prediction of energy transfer efficiency in laser beam welding", Weld. J. 75, 24-s (1996).

[12]. Operation and Maintenance Manual for Surelite Lasers, Continuum, Santa Clara, CA, USA (2002).

[13]. L. Pawlowski, “Thick laser coatings: A review”, J. Therm. Spray Techn. 8, 279-295 (1999).

[14].E. Biro, D. C. Weckman, Y. Zhou, "Pulsed Nd:YAG laser welding of copper using oxygenated assist gases", Metall. Mater. Trans. A 33A, 2019-2030 (2002).

[15].P. Gregory Engleman, A. Kurella, A. Samant, C. A. Blue, N. B. Dahotre, "The application of laser-induced multi-scale surface texturing", JOM-US 57, 46-50 (2005).

[16]. B. Pecholt, M. Vendan, Y. Dong, P. Molian, "Ultrafast laser micromachining of 3C-SiC thin films for MEMS device fabrication", Int. J. Adv. Manuf. Tech. 39, 239-250 (2008).

[17]. A. Gorkiè, D. Kovaèiè, J. Diaci, "Analysis of sonic waves generated during laser engraving of printed circuits", Int. J. Adv. Manuf. Tech. 42, 138-144 (2009).

[18]. L. Orazi, G. Cuccolini, A. Fortunato, G. Tani, "An automated procedure for material removal rate prediction in laser surface micromanufacturing", Int. J. Adv. Manuf. Tech. 46, 163-171 (2010).

[19]. I. E. Saklakoglu, S. Kasman, "Investigation of micro-milling process parameters for surface roughness and milling depth", Int. J. Adv. Manuf. Tech. 54, 567-578 (2011).

\section{Introduction}

The diversity of laser applications in basic research, medicine, and industry, among others, is well known. In the case of industrial applications, marking by laser is one of interest, proof of which can be seen through its applications to plastic [1], glass [2], micro- and nano-machining objects $[3,4]$. Nowadays some commercial systems are available $[5,6]$. For most industrial applications several years ago, there were only four commercial laser models capable of generating enough power for said applications: the YAG and neodymium, the neodymium glass, the ruby, and the carbon dioxide ones. Both the YAG and the carbon dioxide lasers can emit either pulses or a continuous beam, whereas the ruby and the glass ones only emit pulses [7]. Actually, the most popular lasers used in marking systems are the CW laser, the Q-switched Nd:YAG laser, $\mathrm{CO}_{2}$ lasers and fiber lasers.

This paper describes the development of a laser marking system, which consists in the employment of the following: a pulsed Nd:YAG laser; the design of a positioning table machine (PTM) to move the object to be marked; an electronic circuit based on a microcontroller, PC software to process the image desired for printing, as well as a communication protocol to transfer information between PC, microcontroller, Nd:YAG laser and the PTM. Proofs are developed mainly with dry pine and cedar wood.

\section{Development}

This laser marking system relates each pixel of an image shown on any PC screen with each marked point, thereby forming an image on a piece of wood. Additionally, the color intensity of each pixel corresponds to the depth of each point marked (see Fig. 1), as will be explained later.

At the beginning of this research, test materials were limited to those that are feasible to be printed on; consequently, porous materials were eliminated because there would be a lack of uniformity when they were drilled. Different types of wood were selected, as well as a small plaque of plaster and another one of cement. In the end, dry wood proved to be the most suitable to demonstrate our results.

The marking methodology consists in loading an image in the user's PC interface, processing it 




Fig. 1: Transverse view of the plate, showing points with different depth values related to the intensity of their corresponding grade of gray pixels on the image in electronic format.

and sending the information to the microcontroller. Once the processed image data have been transferred, the microcontroller turns on the laser, moves the PTM to the position of the first point to be marked and starts the series of laser pulses according to the pixel's intensity. Having marked the first point, the microcontroller stops the pulses, moves the PTM to the second point, and starts a new series of pulses, and so on (see Fig. 2).
A description of each part composing the entire system follows.

\subsection{The laser}

The laser used in this proposal is a pulsed Nd:YAG laser (neodymium-doped yttrium aluminum garnet). It is a solid-state laser which uses a doping inside neodymium crystals, enabled with yttrium and aluminum oxide $\left(\mathrm{Nd}: \mathrm{Y}_{3} \mathrm{Al}_{5} \mathrm{O}_{12}\right)$, a variety of garnet for the amplification of its characteristic radiation wavelength of $1064 \mathrm{~nm}$. The YAG crystal is employed for pulses of high repeatability, due to its high thermal conductivity that allows it to dispel the generated heat easily [12].

The pulsed Nd:YAG laser employed in this system is of a Continuum Surelite I-10, simultaneously it emits at 1064, 532 and 266 $\mathrm{nm}$. From these three, we only used wavelengths of $1064 \mathrm{~nm}$ for the engraving and $532 \mathrm{~nm}$ for alignment. The laser operates at an adjustable frequency range of 1 to $10 \mathrm{~Hz}$ at $1064 \mathrm{~nm}$ and emits energy of $320 \mathrm{~mJ}$; pulses have Gaussian profiles, 5-7 ns, $6 \mathrm{~mm}$ diameter and an estimated fluence of $1132 \mathrm{~mJ} / \mathrm{cm}^{2}$.

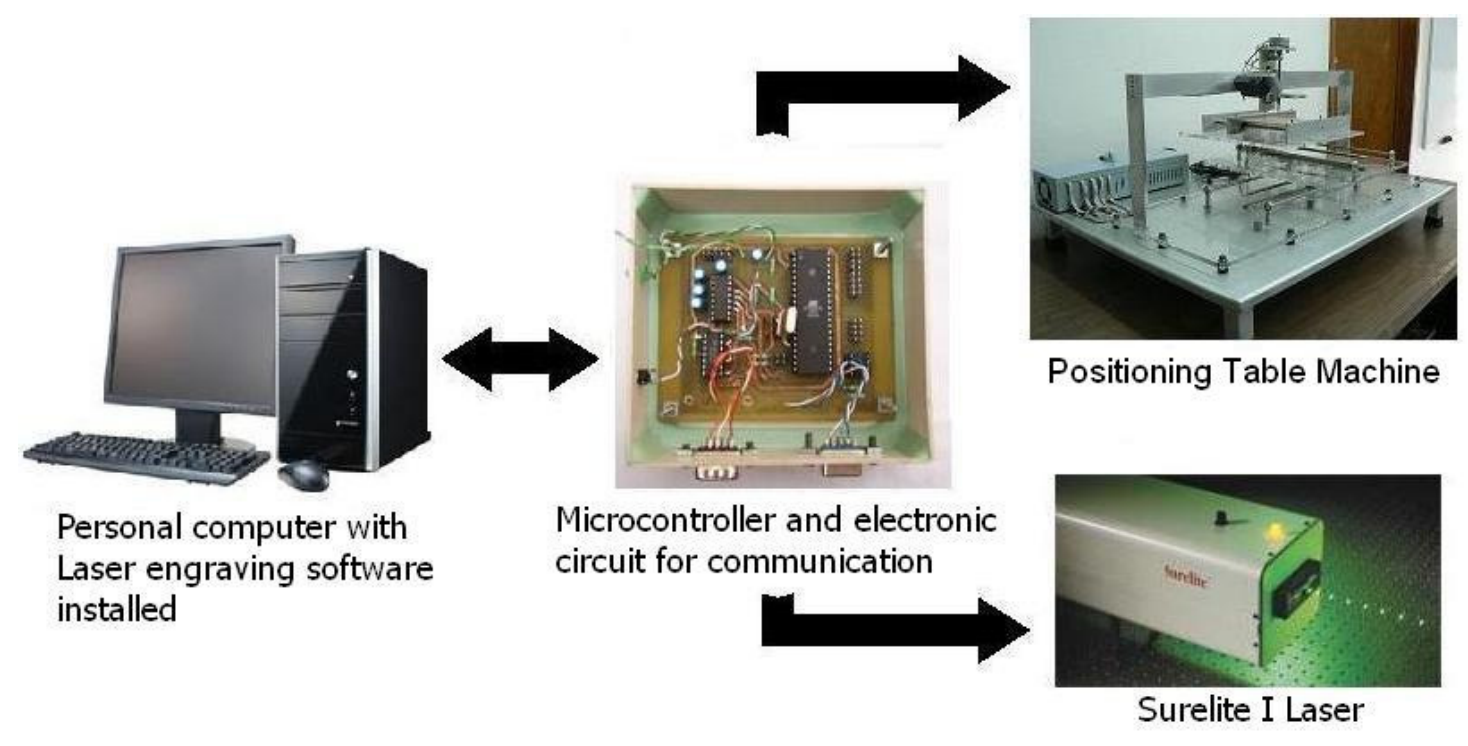

Fig. 2. General diagram of the laser marking system. 


\subsection{Positioning table machine and system description}

Because the laser cannot be manipulated as if it were a pencil, due to its large size and form, it was necessary to design a mechanical system capable of locating any desired point on the imaginary Cartesian plane, on the plate, while the laser remains static. The construction of the positioning table machine (PTM) was based on a tool machine CNC (Computer Numerical Control), previously developed for perforable PCBs (printed circuit boards), see Fig. 3(b). This machine was adapted to become the system that moves the plate to locate each pixel of the image to be printed, while the laser remains static.

At the end, the positioning table machine (PTM) was made up of two parts: the Optical and the Mechanical part. The optical part consists of a fixed lens in a holder, which is focused by a motor. We applied a biconvex lens with a focal length $f=150 \mathrm{~mm}$, see Fig. 3(a). With that, it was possible to control the diameter of the laser pulses, ranging from 0.5 to $6 \mathrm{~mm}$. The mechanical part of this PTM is composed of a sample holder, two screws and two step-by-step motors for moving the sample in a $x y$ plane.

The motors in the $x y$ plane have an angle of $1.8^{\circ}$ and the linear movement produced by the screws is $1.25 \mathrm{~mm}$ for each $360^{\circ}$ rotation. In this way, a resolution of up to 6.25 micrometers (moved with each step of the motor) can be achieved in linear advance. The motor in charge of focusing the lens has a $7.5^{\circ}$ angle and the screw attached to it makes the same progress as the ones in the screw axes. Although this last produces an inaccurate and imprecise linear displacement of the lens with each motor step, it may not be critical for the system's proposal.

The step-by-step motors are controlled through the ports of the microcontroller according with the image data received from the PC. However, these ports do not provide enough energy for their proper operation; therefore a power stage (see Fig. 3(b)) based on power transistors was added and it provides the required energy to the motors.

The image data send to the microcontroller is obtained analyzing the color of each pixel of the image, which is a combination of the three primary colors of light: red, green and blue. Once the amount of each primary color of the pixel is obtained, it is converted to grayscale and saved in an element of a matrix; then the same action is taken with the adjacent pixel. This procedure is performed until the entire image is converted to gray or black and white scale (as it is better known). In this way, an image of $30 \times 50$ pixels will be saved in a matrix of $30 \times 50$ elements, each of them stored in a byte.

Since a byte can take 256 different values (from 0 to 255), there is the same number of intensity values for each pixel: 0 for a white pixel and 255 for a black one. The scale was reduced from 256 to 200 values due to the implemented communication protocol. As an extra tool, the scale can be changed from $0-199$ to $0-50$ according to the user's needs, making the marked points more or less deep.
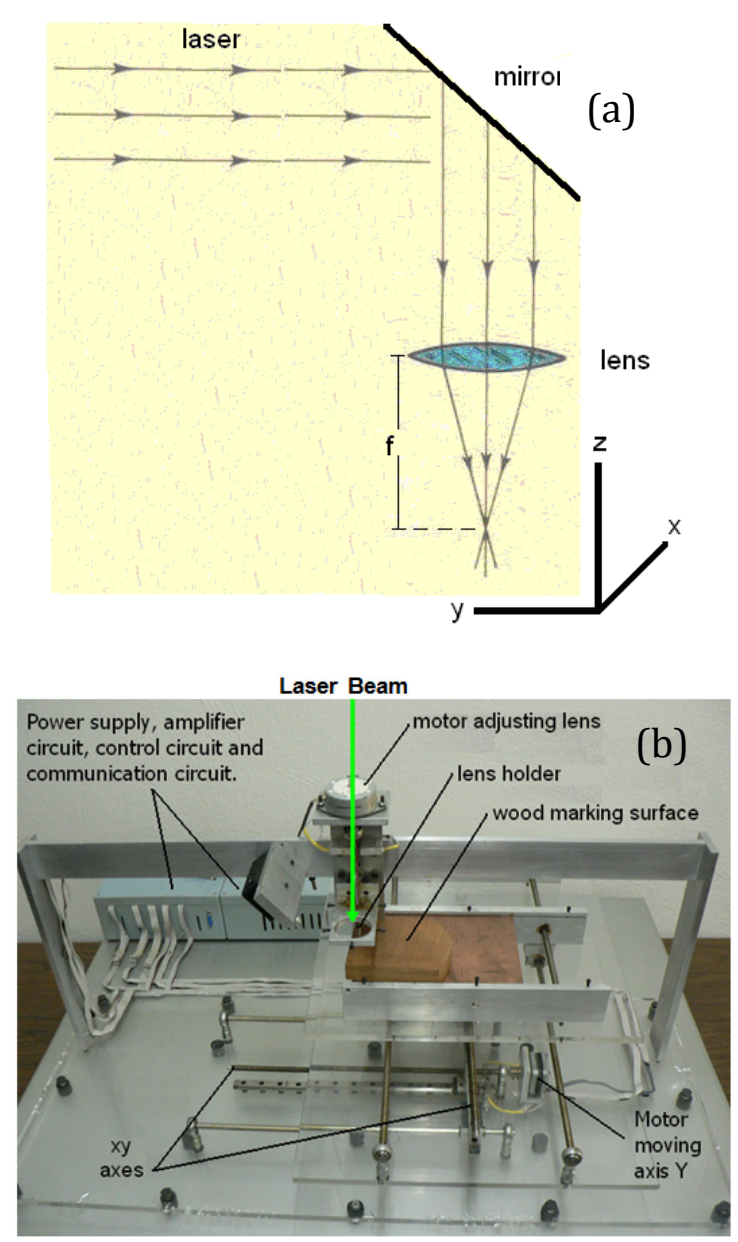

Fig. 3: (a) Marking scheme and, (b) Picture of the tool machine for marking surfaces. Here, sample moves in a $x y$ plane. 
Note that there are certain pixels that are considered as errors in any image editing programs (we will see it in Fig. 6(b)). The intensity of these pixels is very close to the background of the image (usually white), so they are imperceptible to the eye, but these are observed easily with a rapprochement. For such a case, the software includes a filter in order to avoid these errors. This filter works by turning the erroneous pixels into white; in other words, it changes their intensity to zero, eliminating them from the printing. This filter is applied automatically at the time of image processing, and it can be adjusted in the configuration menu of an user's interface, see Fig. 4.

Once the image has been processed, this information is transmitted by RS232 from the PC to the microcontroller and stored in an external EEPROM memory. The microcontroller is also communicated by RS232 with the laser in order to control the marking process (see Fig. 2). Two sets of instructions were designed, one for the
PC and the other for the microcontroller. The control always comes from the PC, this being the one that initiates any transmission; whenever the microcontroller receives a byte, analyzes it, and determines if it is an instruction (byte with value up to 200 in decimal code) or a datum (byte with value less than 200 in decimal code). It takes about 1 minute to transmit the data of an image 100 by 100 pixels in size (that is the maximum size for an image).

For all this actions, a virtual tool for the user's interface was developed in Visual Basic 6.0. The window is shown in Fig. 4 and it has the buttons needed to perform the complete printing routine, such as open the image, modify its dimensions, transmit the image data to the microcontroller and start or stop the marking. The time required to complete the marking process depends on the size of the image. Also a simulation of the printed image is displayed in the right box of the figure.

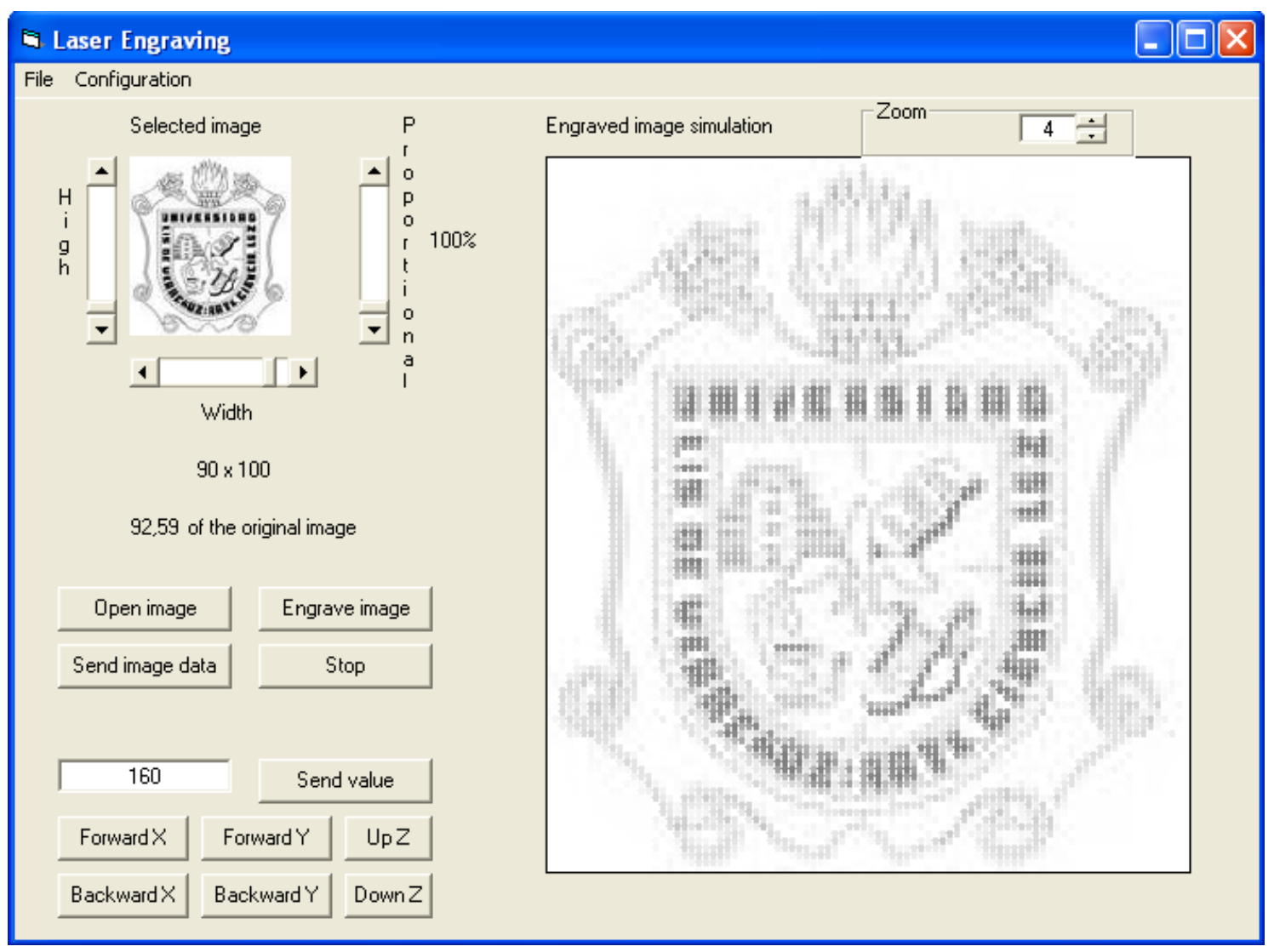

Fig. 4: Main window of the user's interface. A selected image and a processed image for printing are observed. 
If any calibration of the PTM is required, the buttons Move $\mathrm{X}$ and Move $\mathrm{Y}$ have the function of floating over the surface to print along the $X$ and $\mathrm{Y}$ axes, respectively. The Move $\mathrm{Z}$ button zooms the lens in or out from the surface to be printed, thereby controlling the diameter of the incision. The distance that each axis moves is defined by the number of steps specified in the text box located above the mentioned buttons.

\section{Results}

It is no easy to explain the physical phenomena involved in a laser marking process. In general, interaction laser radiation with mater depends of the material as well as the wavelength and power of the light source, but also, if it is coming from a continuum wave $(\mathrm{cw})$ or from a pulsed laser. A revision of all that process is founded for example, in references $[8,9]$. In general, under a $\mathrm{cw}$ or long pulse regimen $(\Delta t>\mathrm{fs})$, interaction thermal processes strongly influence the quality of the resulting feature; in thermal processing, heat is conducted away from the immediate optical absorption region into a wider heataffected zone. In this region, local melting occurs and the ejection of liquid from the interaction region to its edge leads to recast and burr formation. In other hand, in a pulsed interaction regimen, the effect over the matter is fundamentally non-thermal in nature and the intense electric field of the pulses results in strong multi-photon absorption and a shallow, well-defined absorption region. The material is ablated by ionization and is removed as plasma, with the result that there is no heat-affected zone, negligible melting and no burr formation [8].

In our case of study, and according to our standing, the interaction between a pulsed laser and wood would be better explained by a deep penetration welding (or, as it is frequently called, keyhole welding) of the laser radiation in the wood surface. Here, multiple reflections of the beam at the keyhole walls and, when welding with very high power, together with the absorption of radiation by the plasma within the keyhole, the energy coupling may amount to up to $90 \%$ of the incident radiation $[10,11]$. For process fundamentals details see ref. [8].
Now, we present the results obtained from the operation of the proposed laser marking system. The time it takes to print each image depends on its original size, the depth scale assigned and the value for the filtering of undesired pixels. Figure 5(a) shows a test over dry cedar wood, which was not performed using the laser marking system, but was done only to show a relationship between the intensity of a gray pixel in an image and the number of drilling pulses. Thereby, the scale of zero pulse for a "white" point and 255 pulses for a "black" point was established; in all cases a frequency of $10 \mathrm{~Hz}$ was used. The relationship between the number of pulses and their depth was studied and plotted in Fig. 5(b). In this case, the marked point's diameters are approximately $1.2 \mathrm{~mm}$ because our lens was a little out of focus, and we obtained an estimated fluence of $28.2 \mathrm{~J} / \mathrm{cm}^{2}$.

As the number of pulses increases, the depth of the marks increases too, and the material around the point's circumference is also burned, but this last factor does not increase the point's


Fig. 5: (a) Relation between gray intensity of a pixel and number of perforation pulses over cedar wood. (b) Experimental relation between deep of perforation and number of pulses. 
diameter. The drilling process is due to the ablation and burning of the surface material (as we mentioned at the beginning of this section). We can summary all this saying that, in the final product we observed deep penetration welding with some burning, this last probably due to small flue heat ascending to the surface. In spite of this, additional damage to the printed surface was not observed.

The image in Figure 6a was obtained with the laser marking system. Here, a print of the two letters "uv" is shown on a dry cedar wood surface. To perform this work, the image filter (mentioned previously) was not applied. Here, the black points received 199 pulses (because of the communication protocol) from the laser beam, while the clear ones (classified as errors) received between 15 and 30 pulses with a diameter of approximately $1 \mathrm{~mm}$ (with a fluence of $40.7 \mathrm{~J} / \mathrm{cm}^{2}$ ).

Changing some conditions, like reducing the number of pulses between 20 and 30 pulses per point (at $10 \mathrm{~Hz}$ ) and focusing our lens optimally, did not cause any burning of material around the points. Figure 7 shows several tests, developed under these conditions, to print some geometric figures on dry pine wood. The figures printed were a) a rhombus, b) a cross, c) a rotated square, d) a square and e) a straight line.

Also, under the same last conditions, the image in Fig. 8 was obtained; it shows the result

(a)



(b)



Fig. 6: (a) A printing example on a dry cedar wood surface formed by points of $1 \mathrm{~mm}$ diameter approximately, and 255 pulses per point. (b) Undesired spots in the preview digital image. of printing an entire image on a cedar wood surface, with a size reduced to $90 \times 98$ pixel dimensions. The diameter of each point is equal to $0.5 \mathrm{~mm}$ (with a fluence of $162.9 \mathrm{~J} / \mathrm{cm}^{2}$ ). The time spent on the transmission of the processed image data to the microcontroller and their respective backup into EEPROM was 55 seconds. The time elapsed during the engraving was 2 hours and 17 minutes, with a scale from 0 to 50 pulses per point.

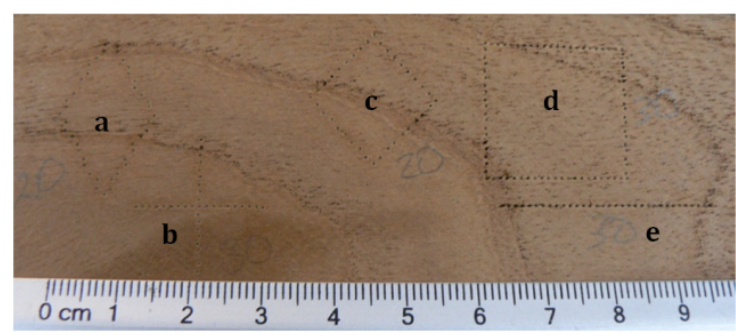

Fig. 7. Geometric figures over pine wood (rhombus, cross, rotated square, square and a straight line). The diameter of each point is equal to $0.5 \mathrm{~mm}$ and we applied 20 to 30 pulses per point.

(a)
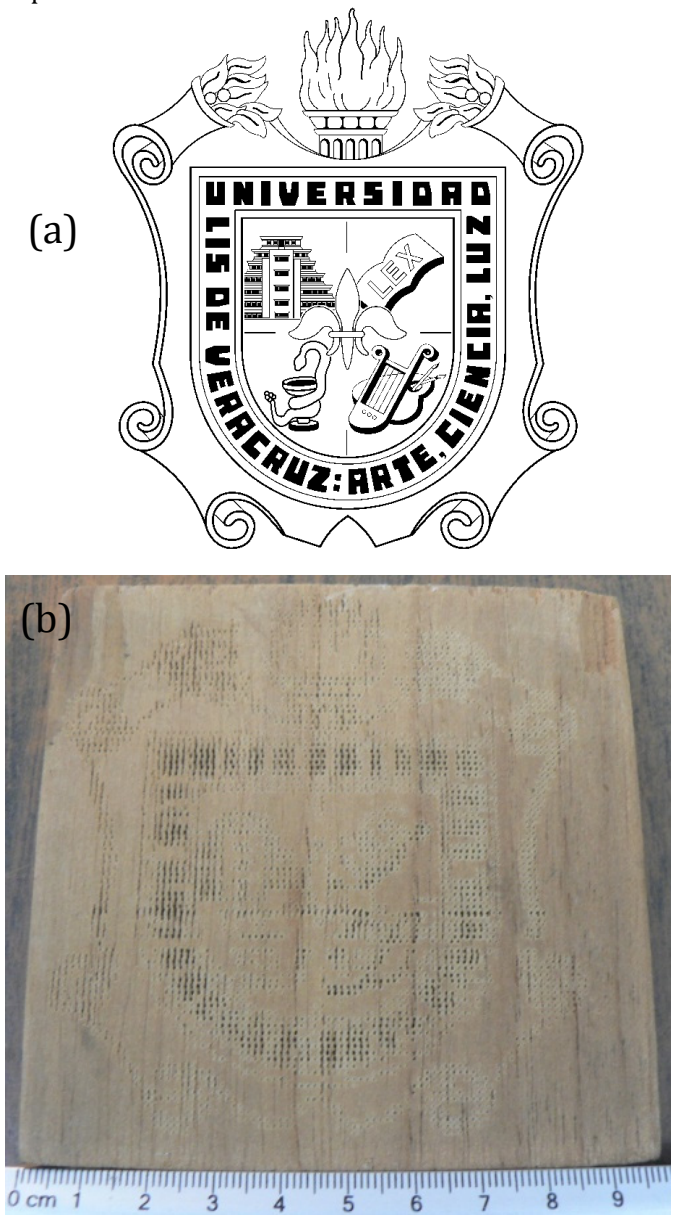

Fig. 8: The Universidad Veracruzana shield. (a) Original image, (b) complete printing of the image. 


\section{Conclusions}

We showed the development and the proof of a complete laser marking system with applications over a dry wood surface, using a standard pulsed $\mathrm{Nd}$ :YAG laser. We were able to print from a single point to a complex image over the mentioned surface.

The following contributions may be attributed to this paper: 1) the proposal of a specific application to wood surface laser marking and printing; 2) as a part of said proposal, the complete design of a communications link system connecting the laser, a microcontroller and a personal computer.

The designed system does not only have the ability to print geometrical figures, but also to outline or sketch an image on an electronic format. More still, it can provide relief engraving, conjugating the colors of the image with the depth of the engraving. The selection, editing and processing of the image, as well as all the details concerning the engraving process are controlled from a PC by means of a graphical user interface. The computer does not control the laser directly; it transfers the processed image information to a microcontroller, which in turn stores the image in an EEPROM memory and controls the PTM and the laser throughout the process of engraving once the order is sent from the computer.
Certainly, the system should be improved, but we have proved that it works well. In the near future, the marking resolution should be improved by increasing the resolution step movement in the motors. Also, this system is open to be tested on other materials. In addition, due to the importance of recent research associated with marking [13-19], the development and the objective of the present work should be taken into consideration for any new applications resulting therefrom.

According to our understanding, there is at present no deep study in relation to the marking of wood surfaces by using pulsed lasers. As we mentioned, the main contributions of this paper are the development of a self-implemented marking system, the use of a specifically pulsed laser and the implementation of a study for marking a wooden surface.

\section{Acknowledgments}

Authors want to tanks the discussions and suggestions for this paper of F.J. GonzálezMartínez and J. Sánchez-Orea. Both, academics of Facultad de Ingeniería en Instrumentación Electrónica, Universidad Veracruzana, Xalapa, Ver. México. 